\title{
CREB3 subfamily transcription factors are not created equal: Recent insights from global analyses and animal models
}

\author{
Chi-Ping Chan, Kin-Hang Kok, Dong-Yan Jin ${ }^{*}$
}

\begin{abstract}
The CREB3 subfamily of membrane-bound bZIP transcription factors has five members in mammals known as CREB3 and CREB3L1-L4. One current model suggests that CREB3 subfamily transcription factors are similar to ATF6 in regulated intramembrane proteolysis and transcriptional activation. Particularly, they were all thought to be proteolytically activated in response to endoplasmic reticulum (ER) stress to stimulate genes that are involved in unfolded protein response (UPR). Although the physiological inducers of their proteolytic activation remain to be identified, recent findings from microarray analyses, RNAi screens and gene knockouts not only demonstrated their critical roles in regulating development, metabolism, secretion, survival and tumorigenesis, but also revealed cell type-specific patterns in the activation of their target genes. Members of the CREB3 subfamily show differential activity despite their structural similarity. The spectrum of their biological function expands beyond ER stress and UPR. Further analyses are required to elucidate the mechanism of their proteolytic activation and the molecular basis of their target recognition.
\end{abstract}

\section{Introduction}

The CREB3 subfamily of bZIP transcription factors in mammals comprises CREB3 (also known as LZIP or Luman), CREB3L1 (OASIS), CREB3L2 (BBF2H7), CREB3L3 (CREB-H) and CREB3L4 (AIbZIP) [1]. CREB3, the prototype of this subfamily, was first identified through its interaction with a transcriptional coactivator termed host cell factor 1 (HCF1) [2]. CREB3L1 was initially found in long-term cultured mouse astrocytes and thought to have a role in gliotic events [3]. CREB3L2 was identified as part of a fusion oncoprotein named FUS-CREB3L2, which was generated by a chromosomal translocation in low grade fibromyxoid sarcoma (LGFMS) [4]. For CREB3L3, it was first reported to be a liver-specific transcription factor [5]. CREB3L4 was originally identified as a highly expressed and androgen-induced protein in prostate cancer cells [6]. The CREB3 subfamily members are closely-related to Drosophila dCREB-A/BBF2 $[7,8]$. They share significant homology within their bZIP domain that mediates

\footnotetext{
* Correspondence: dyjin@hkucc.hku.hk

Department of Biochemistry and State Key Laboratory for Liver Research, LKS Faculty of Medicine, The University of Hong Kong
}

DNA-binding and dimerization [9]. Similar to ATF6, they also feature a transmembrane domain at the immediate C-terminal side of the bZIP region (Figure 1) $[1,10]$. As such, they are type II membrane-associated proteins with the $\mathrm{N}$-terminus facing the cytoplasm and the $\mathrm{C}$-terminus penetrating through the endoplasmic reticulum (ER) membrane into the ER lumen $[11,12]$. Based on their structural similarity to ATF6, transcription factors of the CREB3 subfamily are thought to be activated through regulated intramembrane proteolysis (RIP) in response to ER stress [12]. Upon activation, they are transported from the ER to the Golgi apparatus and proteolytically cleaved there by site 1 protease (S1P) and S2P sequentially to release the $\mathrm{N}$-terminal fragment, which translocates into the nucleus to activate the transcription of genes that play important roles in unfolded protein response (UPR) [12,13]. This model is generally applicable to ATF6 and all CREB3 subfamily transcription factors (Figure 2). In this model, the ER-anchored uncleaved full-length form of the transcription factor remains inactive. Activation through RIP ensures a rapid and timely response to ER stress and UPR $[1,10,14-16]$. RIP is therefore a rate-limiting step in transcription factor activation. In addition to RIP, there are several other 


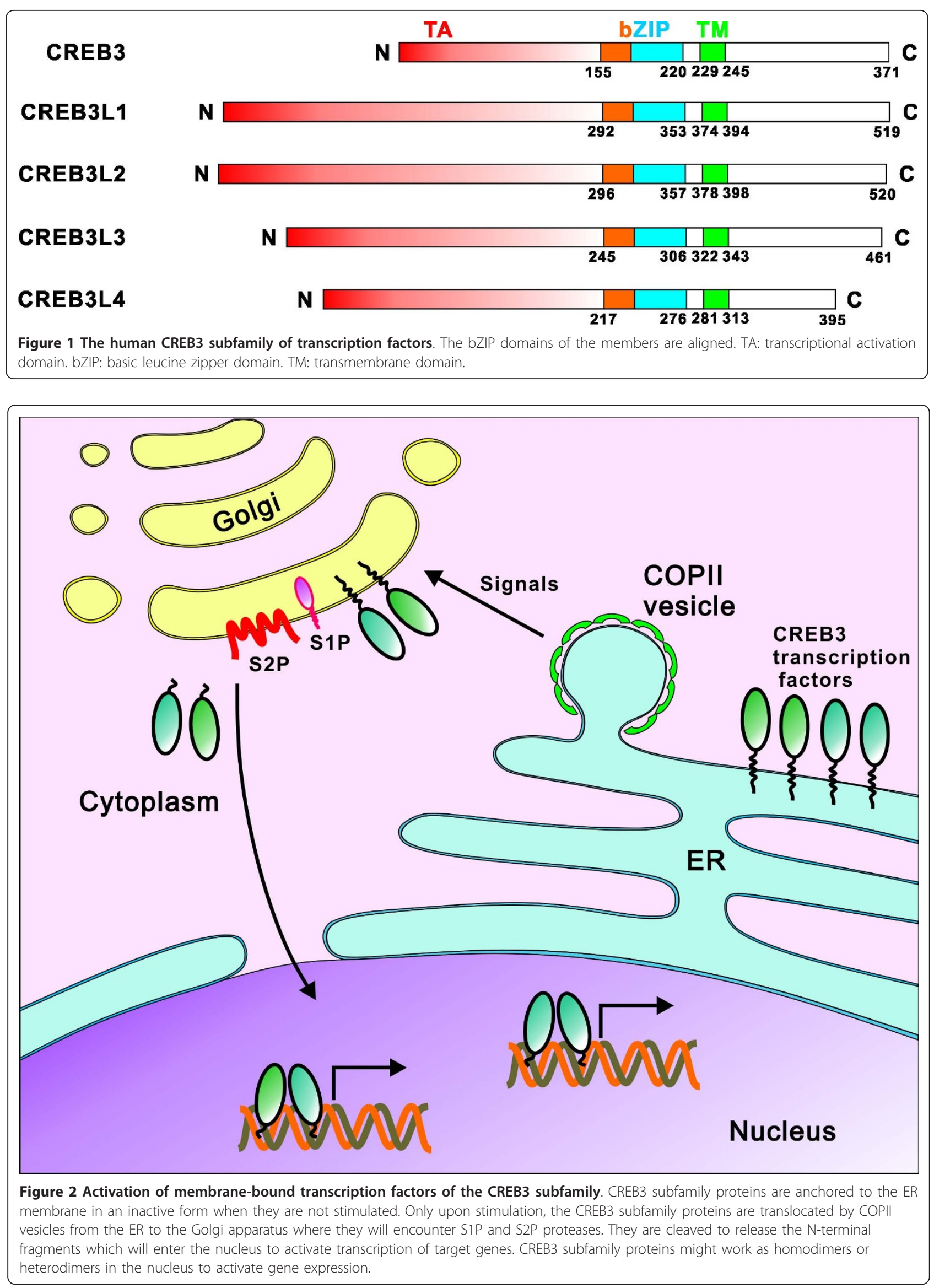


regulatory points. For example, the $\mathrm{N}$-terminal active form of CREB3 subfamily transcription factors can form homo- and hetero-dimers with differential transcriptional activity [9]. Particularly, CREB3L3 was suggested to form a heterodimer with ATF6 to synergistically activate target genes [17]. Whether this heterodimer is thermodynamically stable and functional remains to be determined.

Although it is well accepted that the CREB3 subfamily transcription factors are activated through RIP, the jury is still out for several fundamentally important questions in the model that explains their activation. First, the physiological stimuli for proteolytic activation of discrete transcription factors in the CREB3 subfamily remain to be identified. Particularly, it will be of interest to clarify whether all these transcription factors are activated by ER stress. Second, the physiological targets of these transcription factors should be further characterized. Importantly, whether and how they activate UPR genes and other targets warrant additional analyses. Finally, the physiological roles of different transcription factors in this subfamily merit further investigations. Specifically, it will be of pivotal importance to determine how different factors execute their function in different tissues and cells. In this minireview, we will briefly discuss recent findings that advance our understanding in the above three areas. Collectively, the new evidence supports the notion that the five members in this subfamily have cell type-specific roles. Malfunction of these transcription factors is implicated in different diseases including cancer.

\section{Physiological roles of CREB3 subfamily transcription factors}

One important insight of the CREB3 subfamily transcription factors was derived from Creb3l1 and Creb3l2 knockout mice [18]. Both CREB3L1 and CREB3L2 were previously implicated in UPR in astrocytes or glioma cells. They were shown to undergo RIP under ER stress, leading to the activation of Grp78 [15]. Although hippocampal pyramidal neurons in Creb3 $\mathrm{L1}^{-1-}$ mice were slightly more susceptible to kainic acid, which also induces ER stress [19], the lack of either Grp78 reduction or strong neuronal phenotypes in the knockout mice raised significant concerns about the validity of the earlier data obtained from cultured glioma cells. In addition, one recent study indicated that expression of CREB3L1 in pancreatic $\beta$-cells did not induce Grp78 or other classical UPR genes, but boosted a subset of genes involved in the production of extracellular matrix proteins [20]. Thus, further analyses or re-analyses are required to resolve the discrepancies in the activation of UPR genes by CREB3L1 and CREB3L2.
The new findings from mouse model indicated the requirement of CREB3L1 for bone formation [18]. CREB3L1 was found to be abundantly expressed in osteoblasts. In the Creb3l1 knockout mice, severe osteopenia was observed as the result of a drop in type 1 collagen in the bone matrix together with a decrease in the osteoblast activity. These osteoblasts were found to possess abnormally enlarged rough ER in which a large amount of bone matrix proteins were accumulated. The cause for this phenotype in Creb3l1 ${ }^{-1-}$ osteoblasts is still unclear but it could be due to the lack of activation of CREB3L1 target genes required for transport of bone matrix proteins from ER to Golgi. On the other hand, the type 1 collagen gene Colla1 was identified as a direct target of CREB3L1 whose transactivation is achieved via an enhancer element in the osteoblast-specific Col1a1 promoter. CREB3L1 was essential for bone formation, plausibly by activating Col1a 1 transcription and the secretion of bone matrix proteins [18]. This is the first evidence in support of a role for CREB3L1 in the secretory pathway.

Interestingly, a back-to-back paper on Creb3l2 knockout mice demonstrated a crucial role of CREB3L2 in chondrogenesis [21]. Sec23a, a coat protein complex II (COPII) component for transporting proteins from ER to Golgi, was shown to be a target of CREB3L2. The Creb3l2 $2^{-/}$mice suffered from severe chondrodysplasia and had an immature chest cavity causing death from suffocation within a short time after birth. There were defects in cartilage formation and the abundance of extracellular matrix proteins was prominently reduced. Abnormally enlarged ER, which contains aggregated type II collagen (Col2) and cartilage oligomeric matrix protein (COMP), was observed in the proliferating chondrocytes. These findings supported the model in which CREB3L2 activates the Sec23a-dependent pathway required for secretion of collagens and other extracellular matrix proteins during normal chondrogenesis [21]. This is in keeping with the role of CREB3L1 in bone formation [18]. Together, the two studies pointed to an essential role of CREB3L1 and CREB3L2 in protein secretion. This idea was further supported by two recent studies conducted in other model organisms. In one study, Xenopus CREB3L2 was shown to be required for activation of the secretory pathway during notochord formation [22]. In another more recent report, Drosophila dCREB-A as well as mammalian CREB3L1 and CREB3L2 were found to be major and direct regulators of secretory capacity [23].

CREB3L1 and CREB3L2 are more closely related to each other than to CREB3, CREB3L3 and CREB3L4. The data obtained from animal models raised two possibilities concerning the role of CREB3 subfamily transcription factors in the regulation of protein secretion. In one perspective, 
CREB3L1 and CREB3L2 might serve a specialized regulatory function in secretion, which is evolutionarily conserved in dCREB-A, the single CREB3-like protein in Drosophila [23]. In another model, all five members in the CREB3 subfamily including CREB3, CREB3L3 and CREB3L4 could be influential in the regulation of protein secretion in other cell types. Further investigations are required to clarify these two possibilities.

Consistent with a possible role of CREB3L3 in cell secretion, Creb3l3-knockdown and $\mathrm{Creb}_{3} \mathrm{l}^{-/-}$mice were found to have a defect in the mobilization of acute phase response upon ER stress [17]. CREB3L3 is highly expressed during hepatogenesis. However, neither Creb3l3-knockdown nor Creb3l3 ${ }^{-1-}$ mice showed defects in liver formation [17]. In addition to acute phase response genes such as the ones encoding C-reactive protein and amyloid P-component, several other important genes such as those of hepcidin, phosphoenolpyruvate carboxykinase and glucose 6 phosphatase were also direct targets of CREB3L3 [24,25]. Thus, the physiological function of CREB3L3 extends to iron homeostasis, innate immunity and gluconeogenesis.

Knockout mouse models for Creb3l4 were created by two independent groups. The Creb3l4-deficient mice generated by one group had significantly reduced number of spermatozoa in the epididymis although their fertility was not affected [26]. This observation might be explained by increased apoptosis of meiotic or postmeiotic germ cells [26]. This was also confirmed in the Creb3l4 ${ }^{-1}$ mice made by the other group [27]. Although CREB3 is the prototype of the CREB3 subfamily, a knockout mouse model for CREB3 has not been reported. The physiological roles of CREB3 were deduced primarily through overexpression studies conducted in cultured cells and CREB3 interaction partners identified from yeast two hybrid analyses. Particularly, CREB3 was reported to interact with transcriptional coactivator HCF1 [26], hepatitis C virus core protein [28], CC chemokine receptor 1 [29] and dendritic cell specific transmembrane protein [30]. Whether CREB3 indeed plays a role in chemokine signaling and dendritic cell maturation in vivo awaits the creation of knockout mice.

\section{Physiological activators and targets of CREB3 subfamily transcription factors}

Phenotypic characterization of gene knockdown and knockout mice has not only derived novel insights into the physiological roles of the CREB3 subfamily of transcription factors as detailed above, but also provided important clues to their activators and targets. Particularly, identification of their target genes in knockdown and knockout mice by use of microarray technology and other global approaches has substantially advanced our understanding of this issue. Because some of these activators and targets are cell type-specific, we should take the expression pattern of CREB3 subfamily transcription factors into consideration. Although all proteins in this subfamily have similar domain architecture, they exhibit quite different expression profiles in different tissues: CREB3 is ubiquitous [2]; CREB3L1 is more abundantly expressed in some tissues including pancreas, prostate and bone [18,31]; CREB3L2 can be detected in various tissues and the strongest expression occurs in placenta, lung, spleen, intestine and cartilage [4,21]; CREB3L3 has a liver specific expression [5,17,32]; and CREB3L4 is expressed in different organs such as pancreas, liver, and gonads, yet it is most abundant in prostate epithelial cells $[33,34]$. Thus it is understandable that CREB3 subfamily transcription factors might serve different physiological roles in their target cells by modulating distinct subsets of genes in response to specific stimuli.

Whether ER stress induces proteolytic activation of CREB3 subfamily transcription factors remains controversial. Earlier reports by independent groups suggested that they are proteolytically activated by UPR induced by pharmaceutical agents such as tunicamycin or thapsigargin $[17,18,21]$. On the contrary, no activation of CREB3, CREB3L3 and CREB3L4 was found upon ER stress in several other reports. For example, proteolysis of CREB3 was not seen when UPR is activated $[35,36]$. The same trend also applies to the proteolytic activation of CREB3L3 and CREB3L4, which could not be induced by tunicamycin or other ER stressors $[33,37]$. What would be the direct inducer of the proteolytic activation of CREB3 subfamily transcription factors if ER stress is not? There are three schools of thoughts on this issue. First, some membrane-associated bZIP proteins in plants can be activated by salt stress or heat stress $[38,39]$. From an evolutionary point of view, it will be of interest to see whether mammalian transcription factors of the CREB3 subfamily might also be responsive to similar types of cellular stress. Second, a prototypic membrane-bound transcription factor known as sterol regulatory element binding protein (SREBP) responds to intracellular concentration of cholesterol through an ER-anchored escort protein named SREBP cleavage-activating protein (SCAP) and its regulator Insig [40,41]. Further investigations are required to determine whether similar or distinct escort proteins might also be influential in the activation of CREB3 subfamily transcription factors. Third, the phenotypes of knockout mice and the targets of CREB3 subfamily transcription factors found by global analyses are most revealing in the identification of their physiological activators. For instance, because CREB3L1 and CREB3L2 appear to be critical regulators of protein secretion and are required for bone and cartilage 
formation [18,20-23], they are plausibly activated by physiological inducers of extracellular matrix protein secretion such as transforming growth factor $\beta$ [42]. Likewise, proteolysis of CREB3L3, which plays a role in acute phase response, was found to be induced by interleukin $1 \beta$ and interleukin $6[17,43]$, proinflammatory cytokines that regulate acute phase response. In line with its activation of genes involved in lipid metabolism [17], fatty acids were found to be activators of CREB3L3 $[44,45]$. For CREB3L4, it was found that androgens could induce its expression in a dose-dependent manner [6]. Consistent with a role in dendritic cell maturation, CREB3 was recently shown to be proteolytically activated after stimulation with LPS [30]. Generally, physiological activators of CREB3 subfamily transcription factors are more diverse and not limited to ER stress alone.

CREB3 subfamily transcription factors are thought to recognize cAMP-responsive element (CRE; TGACGTCA), box-B element (TACACGTAATC), ER stress responsive element II (ERSE-II; ATTGG-N-CCACG) and UPR element (UPRE; TGACGTGG) $[2,11,33,46,47]$. Whether different factors prefer a particular enhancer element has not been systematically investigated. Interestingly, microarray analyses revealed that distinct subsets of target genes are activated by CREB3L1, CREB3L2, CREB3L3 and CREB3L4 $[17,18,20,21,23,33]$. For example, CREB3L1 target genes include collagen genes Colla1 and Colla2 as well as other genes implicated in matrix protein production such as Opn, Ocn, Papss2, Matn1 and Chst12 [18,20]. CREB3L2 activates Sec23a, Sec23b and Sec24c genes that are involved in protein secretion [21], it could also turn on a subset of innate immunity-related genes such as MxA [23]. CREB3L3 stimulates Sap and Crp genes that are important in acute phase response, as well as other genes that may affect lipid metabolism [17]. Lastly, ANG, ANKH, $A Q P 9, I D 2$ and KDELR3 genes that are implicated in various cellular processes include protein sorting are regulated by CREB3L4 [33]. Therefore, it will be of particularly great interest to determine whether the different spectra of target genes might be attributed to the cell type and/or the recognition sequence. Because the enhancer sequence recognized by CREB3 subfamily transcription factors has only been characterized in a very limited number of target genes, bioinformatic analysis and experimental validation of the recognition sequence in additional target genes should be performed to clarify whether these transcription factors might recognize other elements. Although some of these target genes might still be pertinent to ER stress and UPR, many are directly involved in other biological processes such as protein secretion, acute phase response, innate immunity and lipid metabolism [17,18,20,21,23,33]. While elucidation of the link between ER stress and these processes would expand our knowledge concerning the biological function of UPR, full characterization of these targets might derive novel insight into the physiological roles of CREB3 subfamily transcription factors beyond ER stress and UPR.

\section{Roles of CREB3 subfamily transcription factors in cellular transformation}

CREB3 subfamily transcription factors exist ambiently in an inactive form bound to the ER membrane. The active form is usually short-lived and rapidly degraded by the proteosome [32,37]. Plausibly, constitutive activation of these transcription factors might have deleterious effects that are either tumor suppressive or oncogenic in different contexts. For example, whereas the proapoptotic activity might suppress oncogenesis, aberrant stimulation of extracellular matrix protein secretion could promote tumor formation and metastasis. It is therefore not surprising that all five members of the CREB3 subfamily have been implicated in cellular transformation. Below we will briefly review the roles of individual members in different types of cancer.

CREB3 was known to be a binding partner of hepatitis $C$ virus core protein. This interaction with the viral oncoprotein might interfere with a tumor suppressive function of CREB3 to promote cellular transformation in hepatocellular carcinoma [28]. CREB3 was also reported to be influential in the metastasis of breast cancer cells [48]. CREB3L1 and CREB3L2 are fusion partners of FUS in the generation of chimeric oncoprotein by chromosomal translocation in a soft tissue sarcoma known as LGFMS $[1,4,49,50]$. The FUSCREB3L1 or FUS-CREB3L2 fusion oncoproteins include the $\mathrm{N}$-terminal fragment of FUS and the $\mathrm{C}$ terminal fragment of CREB3L1 or CREB3L2 harboring the bZIP domain [4]. FUS-CREB3L2 has a very high incidence of $96 \%$ in successfully evaluated LGFMS cases whereas FUS-CREB3L1 was only found in one exceptional case of LGFMS [49]. Since expression of FUS-CREB3L2 is under the control of the strong FUS promoter, FUS-CREB3L2 is generally thought to be overexpressed in LGFMS. Additionally, the $\mathrm{N}$-terminal part of FUS also contributes transactivating and oncogenic properties as in the case of other FUS-containing fusion oncoproteins [51]. Thus, FUS-CREB3L2 is a hyperactive transcription factor that turns on the expression of various target genes. Consistent with a role of CREB3L2 in stimulating the secretion of extracellular matrix proteins [18,23], aberrant accumulation of collagens was observed in LGFMS [52]. In this regard, it will be of great interest to elucidate how overproduction of collagen might underlie the pathology of LGFMS. In further support of the oncogenic property of CREB3L2, it was also found to be involved in another chromosomal translocation in thyroid 
carcinoma that gives rise to the CREB3L2-PPAR $\gamma$ fusion oncogene [53]. The endocrine function of the thyroid gland prompted us to the hypothesis that CREB3L2-PPAR $\gamma$ might also exert an impact on protein secretion in thyroid carcinoma. Further investigations are needed to test this hypothesis. Finally, a recent RNAi screen revealed a role for CREB3L2 in the survival pathway in malignant glioma cells. This was mediated through ATF5 and an ATF5 target known as myeloid cell leukemia sequence-1 (MCL1). The transcription of ATF5 was activated by CREB3L2, which was induced by Ras-mitogen-activated protein kinase or phosphoinositide-3-kinase signaling pathway. Taken together, these findings revealed an antiapoptotic activity of CREB3L2 and provided a new mechanism to explain the uncontrolled proliferation of malignant glioma cells [54]. CREB3L3 was found to be underexpressed in hepatocellular carcinoma and to serve a growth suppressive role in hepatocytes [32]. In this regard, it will be intriguing to see whether the $\mathrm{Creb}_{3} \mathrm{l}^{-/-}$mice might be more susceptible to liver cancer. Finally, the higher abundance of CREB3L4 in prostate cancer cells and its inducibility by androgens are in line with a crucial role for CREB3L4 in prostate carcinogenesis $[6,34]$. Nevertheless, further elucidation of the roles of discrete transcription factors in the CREB3 subfamily in cellular transformation might derive new approaches for rational design of targeted anti-cancer agents.

\section{Conclusions}

The CREB3 subfamily transcription factors are not only involved in ER stress and UPR as previously envisaged, but also play a crucial role in many other biological processes including cell secretion, bone and cartilage formation as well as oncogenesis. Understanding the physiological activators and targets of this subfamily by use of a combination of global unbiased analyses, individual gene approaches and animal models will derive new knowledge in transcriptional regulation and new strategies in the intervention of human diseases.

\section{Acknowledgements}

Research in our laboratory was supported by grants from the Hong Kong Research Grants Council (HKU 7486/06M, HKU 1/06C, HKU 7661/08M, N_HKU 720/08 and HKU 7/CRF/09).

\section{Authors' contributions}

CPC, KHK and DYJ wrote the review. All authors read and approved the final manuscript.

\section{Competing interests}

The authors declare that they have no competing interests.

Received: 4 January 2011 Accepted: 17 February 2011 Published: 17 February 2011
References

1. Panagopoulos I, Moller E, Dahlen A, Isaksson M, Mandahl N, VlamisGardikas A, Mertens F: Characterization of the native CREB3L2 transcription factor and the FUS/CREB3L2 chimera. Genes Chromosomes Cancer 2007, 46:181-191.

2. Lu R, Yang P, O'Hare P, Misra V: Luman, a new member of the CREB/ATF family, binds to herpes simplex virus VP16-associated host cellular factor. Mol Cell Biol 1997, 17:5117-5126.

3. Honma Y, Kanazawa K, Mori T, Tanno Y, Tojo M, Kiyosawa H, Takeda J, Nikaido T, Tsukamoto T, Yokoya S, Wanaka A: Identification of a novel gene, OASIS, which encodes for a putative CREB/ATF family transcription factor in the long-term cultured astrocytes and gliotic tissue. Brain Res Mol Brain Res 1999, 69:93-103.

4. Storlazzi CT, Mertens F, Nascimento A, Isaksson M, Wejde J, Brosjo O, Mandahl N, Panagopoulos I: Fusion of the FUS and BBF2H7 genes in low grade fibromyxoid sarcoma. Hum Mol Genet 2003, 12:2349-2358.

5. Omori Y, Imai J, Watanabe M, Komatsu T, Suzuki Y, Kataoka K, Watanabe S, Tanigami A, Sugano S: CREB-H: a novel mammalian transcription factor belonging to the CREB/ATF family and functioning via the box-B element with a liver-specific expression. Nucleic Acids Res 2001, 29:2154-2162.

6. Qi H, Fillion C, Labrie Y, Grenier J, Fournier A, Berger L, El-Alfy M, Labrie C: AlbZIP, a novel bZIP gene located on chromosome 1q21.3 that is highly expressed in prostate tumors and of which the expression is upregulated by androgens in LNCaP human prostate cancer cells. Cancer Res 2002, 62:721-733.

7. Smolik SM, Rose RE, Goodman RH: A cyclic AMP-responsive elementbinding transcriptional activator in Drosophila melanogaster, dCREB-A, is a member of the leucine zipper family. Mol Cell Biol 1992, 12:4123-4131.

8. Abel T, Bhatt R, Maniatis T: A Drosophila CREB/ATF transcriptional activator binds to both fat body- and liver-specific regulatory elements. Genes Dev 1992, 6:466-480.

9. Vinson C, Acharya A, Taparowsky EJ: Deciphering B-ZIP transcription factor interactions in vitro and in vivo. Biochim Biophys Acta 2006, 1759:4-12.

10. Stirling J, O'Hare P: CREB4, a transmembrane bZip transcription factor and potential new substrate for regulation and cleavage by S1P. Mol Biol Cell 2006, 17:413-426.

11. DenBoer LM, Hardy-Smith PW, Hogan MR, Cockram GP, Audas TE, Lu R: Luman is capable of binding and activating transcription from the unfolded protein response element. Biochem Biophys Res Commun 2005, 331:113-119.

12. Brown MS, Ye J, Rawson RB, Goldstein JL: Regulated intramembrane proteolysis: a control mechanism conserved from bacteria to humans. Cell 2000, 100:391-398.

13. Bailey D, O'Hare P: Transmembrane bZIP transcription factors in ER stress signaling and the unfolded protein response. Antioxid Redox Signal 2007, 9:2305-2321.

14. Raggo C, Rapin N, Stirling J, Gobeil P, Smith-Windsor E, O'Hare P, Misra V: Luman, the cellular counterpart of herpes simplex virus VP16, is processed by regulated intramembrane proteolysis. Mol Cell Biol 2002, 22:5639-5649.

15. Kondo S, Murakami T, Tatsumi K, Ogata M, Kanemoto S, Otori K, Iseki K, Wanaka A, Imaizumi K: OASIS, a CREB/ATF-family member, modulates UPR signalling in astrocytes. Nat Cell Biol 2005, 7:186-194.

16. Kondo S, Saito A, Hino S, Murakami T, Ogata M, Kanemoto S, Nara S, Yamashita A, Yoshinaga $\mathrm{K}$, Hara $\mathrm{H}$, Imaizumi $\mathrm{K}$ : BBF2H7, a novel transmembrane bZIP transcription factor, is a new type of endoplasmic reticulum stress transducer. Mol Cell Biol 2007, 27:1716-1729.

17. Zhang K, Shen X, Wu J, Sakaki K, Saunders T, Rutkowski DT, Back SH, Kaufman RJ: Endoplasmic reticulum stress activates cleavage of $\mathrm{CREBH}$ to induce a systemic inflammatory response. Cell 2006, 124:587-599.

18. Murakami T, Saito A, Hino S, Kondo S, Kanemoto S, Chihara K, Sekiya H, Tsumagari K, Ochiai K, Yoshinaga K, et al: Signalling mediated by the endoplasmic reticulum stress transducer OASIS is involved in bone formation. Nat Cell Biol 2009, 11:1205-1211.

19. Chihara K, Saito A, Murakami T, Hino S, Aoki Y, Sekiya H, Aikawa Y, Wanaka A, Imaizumi K: Increased vulnerability of hippocampal pyramidal neurons to the toxicity of kainic acid in OASIS-deficient mice. $J$ Neurochem 2009, 110:956-965.

20. Vellanki RN, Zhang L, Guney MA, Rocheleau JV, Gannon M, Volchuk A: OASIS/CREB3L1 induces expression of genes involved in extracellular 
matrix production but not classical endoplasmic reticulum stress response genes in pancreatic $\beta$-cells. Endocrinology 2010, 151:4146-4157.

21. Saito A, Hino S, Murakami T, Kanemoto S, Kondo S, Saitoh M, Nishimura R, Yoneda T, Furuichi T, Ikegawa S, et al: Regulation of endoplasmic reticulum stress response by a BBF2H7-mediated Sec23a pathway is essential for chondrogenesis. Nat Cell Biol 2009, 11:1197-1204.

22. Tanegashima K, Zhao H, Rebbert ML, Dawid IB: Coordinated activation of the secretory pathway during notochord formation in the Xenopus embryo. Development 2009, 136:3543-3548.

23. Fox RM, Hanlon CD, Andrew DJ: The CrebA/Creb3-like transcription factors are major and direct regulators of secretory capacity. J Cell Biol 2010, 191:479-492.

24. Lee MW, Chanda D, Yang J, Oh H, Kim SS, Yoon YS, Hong S, Park KG, Lee IK, Choi CS, et al: Regulation of hepatic gluconeogenesis by an ERbound transcription factor, CREBH. Cell Metab 2010, 11:331-339.

25. Vecchi C, Montosi G, Zhang K, Lamberti I, Duncan SA, Kaufman RJ, Pietrangelo A: ER stress controls iron metabolism through induction of hepcidin. Science 2009, 325:877-880.

26. Adham IM, Eck TJ, Mierau K, Muller N, Sallam MA, Paprotta I, Schubert S, Hoyer-Fender S, Engel W: Reduction of spermatogenesis but not fertility in Creb3l4-deficient mice. Mol Cell Biol 2005, 25:7657-7664

27. Nagamori I, Yomogida K, Ikawa M, Okabe M, Yabuta N, Nojima H: The testes-specific bZip type transcription factor Tisp40 plays a role in ER stress responses and chromatin packaging during spermiogenesis. Genes Cells 2006, 11:1161-1171.

28. Jin DY, Wang HL, Zhou Y, Chun AC, Kibler KV, Hou YD, Kung H, Jeang KT: Hepatitis C virus core protein-induced loss of LZIP function correlates with cellular transformation. EMBO J 2000, 19:729-740.

29. Ko J, Jang SW, Kim YS, Kim IS, Sung HJ, Kim HH, Park JY, Lee YH, Kim J, $\mathrm{Na}$ DS: Human LZIP binds to CCR1 and differentially affects the chemotactic activities of CCR1-dependent chemokines. FASEB J 2004, 18:890-892.

30. Eleveld-Trancikova D, Sanecka A, van Hout-Kuijer MA, Looman MW, Hendriks IA, Jansen BJ, Adema GJ: DC-STAMP interacts with ER-resident transcription factor LUMAN which becomes activated during DC maturation. Mol Immunol 2010, 47:1963-1973.

31. Omori Y, Imai J, Suzuki Y, Watanabe S, Tanigami A, Sugano S: OASIS is a transcriptional activator of CREB/ATF family with a transmembrane domain. Biochem Biophys Res Commun 2002, 293:470-477.

32. Chin KT, Zhou HJ, Wong CM, Lee JM, Chan CP, Qiang BQ, Yuan JG, Ng IO, Jin DY: The liver-enriched transcription factor CREB-H is a growth suppressor protein underexpressed in hepatocellular carcinoma. Nucleic Acids Res 2005, 33:1859-1873.

33. Ben Aicha S, Lessard J, Pelletier M, Fournier A, Calvo E, Labrie C: Transcriptional profiling of genes that are regulated by the endoplasmic reticulum-bound transcription factor AlbZIP/CREB3L4 in prostate cells. Physiol Genomics 2007, 31:295-305.

34. Labrie C, Lessard J, Ben Aicha S, Savard MP, Pelletier M, Fournier A, Lavergne E, Calvo E: Androgen-regulated transcription factor AlbZIP in prostate cancer. J Steroid Biochem Mol Biol 2008, 108:237-244.

35. Chen X, Shen J, Prywes R: The luminal domain of ATF6 senses endoplasmic reticulum (ER) stress and causes translocation of ATF6 from the ER to the Golgi. J Biol Chem 2002, 277:13045-13052.

36. Nadanaka S, Okada T, Yoshida H, Mori K: Role of disulfide bridges formed in the luminal domain of ATF6 in sensing endoplasmic reticulum stress. Mol Cell Biol 2007, 27:1027-1043.

37. Chan CP, Mak TY, Chin KT, Ng IO-L, Jin DY: N-linked glycosylation is required for optimal proteolytic activation of membrane-bound transcription factor CREB-H. J Cell Sci 2010, 123:1438-1448,

38. Gao H, Brandizzi F, Benning C, Larkin RM: A membrane-tethered transcription factor defines a branch of the heat stress response in Arabidopsis thaliana. Proc Natl Acad Sci USA 2008, 105:16398-16403.

39. Liu JX, Srivastava R, Che P, Howell SH: Salt stress responses in Arabidopsis utilize a signal transduction pathway related to endoplasmic reticulum stress signaling. Plant J 2007, 51:897-909.

40. Hua X, Nohturfft A, Goldstein JL, Brown MS: Sterol resistance in $\mathrm{CHO}$ cells traced to point mutation in SREBP cleavage-activating protein. Cell 1996, 87:415-426.

41. Yang T, Espenshade PJ, Wright ME, Yabe D, Gong Y, Aebersold R, Goldstein JL, Brown MS: Crucial step in cholesterol homeostasis: sterols promote binding of SCAP to INSIG-1, a membrane protein that facilitates retention of SREBPs in ER. Cell 2002, 110:489-500.

42. Wu MY, Hill CS: TGF- $\beta$ superfamily signaling in embryonic development and homeostasis. Dev Cell 2009, 16:329-343.

43. Bailey D, Barreca C, O'Hare P: Trafficking of the bZIP transmembrane transcription factor CREB-H into alternate pathways of ERAD and stressregulated intramembrane proteolysis. Traffic 2007, 8:1796-1814.

44. Danno H, Ishii KA, Nakagawa Y, Mikami M, Yamamoto T, Yabe S, Furusawa M, Kumadaki S, Watanabe K, Shimizu H, et al: The liver-enriched transcription factor CREBH is nutritionally regulated and activated by fatty acids and PPARalpha. Biochem Biophys Res Commun 2010, 391:1222-1227

45. Gentile $C L$, Wang D, Pfaffenbach $K T$, Cox R, Wei Y, Pagliassotti MJ: Fatty acids regulate CREBh via transcriptional mechanisms that are dependent on proteasome activity and insulin. Mol Cell Biochem 2010, 344:99-107.

46. Audas TE, Li Y, Liang G, Lu R: A novel protein, Luman/CREB3 recruitment factor, inhibits Luman activation of the unfolded protein response. Mol Cell Biol 2008, 28:3952-3966.

47. Jang SW, Kim YS, Kim YR, Sung HJ, Ko J: Regulation of human LZIP expression by NF-KB and its involvement in monocyte cell migration induced by Lkn-1. J Biol Chem 2007, 282:11092-11100.

48. Kim HC, Choi KC, Choi HK, Kang HB, Kim MJ, Lee YH, Lee OH, Lee J, Kim YJ, Jun W, et al: HDAC3 selectively represses CREB3-mediated transcription and migration of metastatic breast cancer cells. Cell Mol Life Sci 2010, 67:3499-3510

49. Mertens F, Fletcher CD, Antonescu CR, Coindre JM, Colecchia M, Domanski HA, Downs-Kelly E, Fisher C, Goldblum JR, Guillou L, et al: Clinicopathologic and molecular genetic characterization of low-grade fibromyxoid sarcoma, and cloning of a novel FUS/CREB3L1 fusion gene. Lab Invest 2005, 85:408-415

50. Panagopoulos I, Storlazzi CT, Fletcher CD, Fletcher JA, Nascimento A, Domanski HA, Wejde J, Brosjo O, Rydholm A, Isaksson M, et al: The chimeric FUS/CREB312 gene is specific for low-grade fibromyxoid sarcoma. Genes Chromosomes Cancer 2004, 40:218-228.

51. Shing DC, McMullan DJ, Roberts P, Smith K, Chin SF, Nicholson J, Tillman RM, Ramani P, Cullinane C, Coleman N: FUS/ERG gene fusions in Ewing's tumors. Cancer Res 2003, 63:4568-4576.

52. Franchi A, Massi $D$, Santucci M: Hyalinizing spindle cell tumor with giant rosettes and low-grade fibromyxoid sarcoma: an immunohistochemical and ultrastructural comparative investigation. Ultrastruct Pathol 2003, 27:349-355.

53. Lui WO, Zeng L, Rehrmann V, Deshpande S, Tretiakova M, Kaplan EL, Leibiger I, Leibiger B, Enberg U, Hoog A, et al: CREB3L2-PPARy fusion mutation identifies a thyroid signaling pathway regulated by intramembrane proteolysis. Cancer Res 2008, 68:7156-7164.

54. Sheng Z, Li L, Zhu LJ, Smith TW, Demers A, Ross AH, Moser RP, Green MR: A genome-wide RNA interference screen reveals an essential CREB3L2ATF5- MCL1 survival pathway in malignant glioma with therapeutic implications. Nat Med 2010, 16:671-677.

doi:10.1186/2045-3701-1-6

Cite this article as: Chan et al:: CREB3 subfamily transcription factors are not created equal: Recent insights from global analyses and animal models. Cell \& Bioscience 2011 1:6.

\section{Submit your next manuscript to BioMed Central and take full advantage of:}

- Convenient online submission

- Thorough peer review

- No space constraints or color figure charges

- Immediate publication on acceptance

- Inclusion in PubMed, CAS, Scopus and Google Scholar

- Research which is freely available for redistribution 